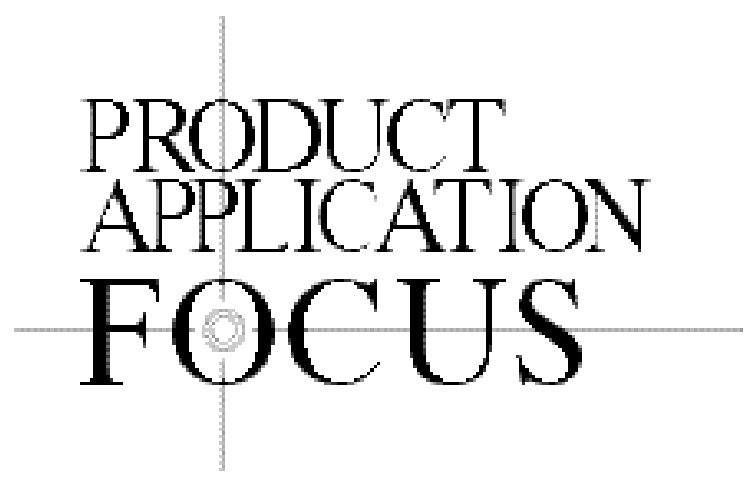

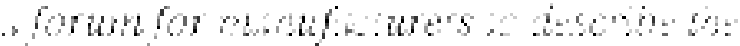

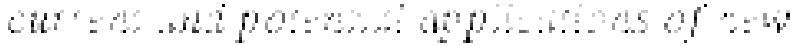

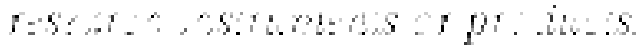

\title{
High-Yield, In Vitro Protein Expression Using a Continuous-Exchange, Coupled Transcription/ Translation System
}

\author{
G.A. Martin, R. Kawaguchi², Y. Lam', A. DeGiovanni, M. Fukushima², \\ and W. Mutter ${ }^{3}$ \\ Roche Diagnostics, Berkeley, CA, ${ }^{1}$ University of California, Berkeley, CA, USA, \\ ${ }^{2}$ SRL, Inc., Hino Japan, and ${ }^{3}$ Roche Diagnostics GmbH, Penzberg, Germany
}

BioTechniques 31:948-953 (October 2001)

\begin{abstract}
The Rapid Translation System (RTS 500) (Roche Molecular Biochemicals) is a high-yield protein expression system that utilizes an enhanced $\mathrm{E}$. coli lysate for an in vitro transcription/translation reaction. In contrast to conventional transcription/translation, this system allows protein expression to continue for more than $24 \mathrm{~h}$. We demonstrated the utility of the RTS 500 by expressing different soluble and active proteins that generally pose problems in cell-based expression systems. We first expressed GFP-lunasin, a fusion protein that, because of its toxicity, has been impossible to produce in whole cells. The second protein we expressed, human interleukin-2 (IL-2), is generally difficult to produce, either as the native molecule or as a GST fusion protein, in a soluble form in bacteria. Finally, we demonstrated the capacity of the RTS 500 to co-express proteins, by the simultaneous production of GFP and CAT in a single reaction. This new technology appears to be particularly useful for the convenient production of preparative amounts $(100-900 \mu \mathrm{g})$ of proteins that are toxic or insoluble in cell-based systems.
\end{abstract}

\section{INTRODUCTION}

For many years, in vitro transcription/translation has been widely used for the synthesis of analytical amounts of proteins. In vitro translation bypasses many aspects of cellular function, thereby offering potential advantages over cellbased expression, especially when producing cytotoxic, unstable, or insoluble proteins. Another advantage of cell-free translation systems versus cell-based systems is that various kinds of modified "unnatural" amino acid labels can be incorporated into proteins efficiently and at a low cost. Further- more, by eliminating the need to manipulate whole cells in culture, in vitro translation systems would be highly amenable for the automated, high-throughput expression of large numbers of proteins. Despite these potential benefits, the very low productivity (usually less than $30 \mu \mathrm{g}$ protein $/ \mathrm{mL}$ reaction) (1) of other commercial cell-free protein expression systems limits the applicability of these systems. Consequently, they are unfeasible alternatives to cell-based expression. Current commercially available in vitro translation systems utilize mRNA and lysates from prokaryotic (E. coli) or eukaryotic (wheat germ or rabbit reticulocyte) sources to supply all necessary components for transcription/translation. The reactions contain limited quantities of small molecular substrates and energy sources and drive expression only for 2-3 $\mathrm{h}$, resulting in low total protein yields.

The hurdle of limited small-molecular-weight substrate supplies and energy sources was overcome either by improving energy regeneration systems (15) or by continuously replenishing low-molecular-weight substrates and removing inhibitory reaction products $(2,19)$. The latter modification extends the in vitro transcription/translation reaction for periods of more than $24 \mathrm{~h}$, increasing yields of protein to hundreds of micrograms per $\mathrm{mL}$ of reaction. Others have reproduced and convincingly demonstrated the merits of this modified technology $(5,6,12-14)$. The Rapid Translation System (RTS 500) incorporates these advancements and enables the rapid production of preparative quantities of protein.

The RTS 500 uses a small, two-compartment device for the transcription/translation reaction (Figure 1). Transcription and translation (based on a modified E. coli lysate) occur within the reaction compartment ( $1 \mathrm{~mL}$ volume). The feeding compartment (10 $\mathrm{mL}$ volume), separated from the reaction 
compartment by a semi-permeable membrane $(10 \mathrm{kDa}$ cutoff), contains a large supply of energy components and amino acids. The concentration of these small molecules has been extensively optimized for maximal performance of the system. Small molecules freely diffuse across this semi-perm eable membrane. Inhibitory reaction end products from the reaction compartment diffuse down the concentration gradient into the feeding compartment. By this same mechanism, essential substrates are replenished into the reaction chamber as they are utilized. The high yields of protein from the RTS 500 now make in vitro transcription/translation a possible alternative to cell-based methods for the production of preparative amounts of recombinant protein $(10,17,20)$.

We performed three challenging protein expression experiments to investigate the capabilities of the RTS 500. In the first experiment, we tested whether it was possible to express a toxic protein. Lunasin, a toxic polypeptide derived from the small subunit of soybean cotyledon-specific $2 \mathrm{~S}$ albumin, prevents cell division in mammalian cells and causes aberrant cell division in bacteria (6). Because of these characteristics, lunasin has been impossible to produce, as either a polypeptide or a GFP-fusion protein, in sufficient quantities, using cell-based systems. In the second set of experiments, we investigated whether it would be possible to express soluble and functional human interleukin-2 (IL-2) with the RTS 500 because IL-2 often forms insoluble aggregates in $E$. coli $(8,9,21)$. The ability to co-express two or more genes is of great interest for many applications, including protein-protein interaction studies; therefore, we tested the simultaneous expression of green fluorescent protein (GFP) and chloramphenicol acetyltransferase (CAT) in the RTS 500.

\section{MATERIALS AND METHODS}

All components of the Rapid Translation System, consisting of the RTS 500 Instrument and the RTS 500 E. coli Circular Template Kit, were provided by Roche Molecular Biochemicals (Indianapolis, IN, USA). All standard expression experiments in the RTS 500 were performed according to the supplier's instructions. The lyophilized components supplied in the RTS 500 E. coli Circular Template Kit were reconstituted and mixed according to the manufacturer's instructions (Figure 1). The two compartments of the RTS 500 reaction device were filled with the appropriate solutions, and the reaction was run in the RTS 500 instrument. Reaction conditions were varied for optimized expression of human recom binant IL-2. The investigated parameters include plasmid amount $(5-20 \mu \mathrm{g})$, reaction temperature $\left(25^{\circ} \mathrm{C}-37^{\circ} \mathrm{C}\right)$, and time of expression (2-24 h) (Figure 3, A and B). Optimized reaction conditions were as follows: $15 \mu \mathrm{g}$ plasmid DNA, with a stirring rate of $120 \mathrm{rpm}$, at $30^{\circ} \mathrm{C}$ for $24 \mathrm{~h}$. Standard reaction conditions, as described in the kit instructions, were used for the expression of GFP-lunasin and the co-expression studies with GFP and CAT.

\section{Cloning}

The pIVEX family of expression plasmids has been optimized for use with the RTS 500 and is included in the RTS 500 E. coli Circular Template Kit. The plasmids include a T7 promoter, T7 terminator, and flanking multiple cloning sites. Both
$\mathrm{N}$ - and $\mathrm{C}$-terminal hexa-his containing vectors are supplied, as well as a plasmid-encoding wild-type GFP (positive control). The plasmid sequences and maps of pIVEX are available at http://proteinexpression.com. The expression plasmid for GFPlunasin was constructed by inserting a cDNA, which encodes a fusion protein, between GFP and lunasin (6). This complex was then cloned into pIVEX 2.4a, between the NcoI and SmaI sites. Similarly, the PCR-generated DNA fragment, containing the mature human IL-2 coding region, was cloned between the NotI and PstI sites of pIVEX2.4a. The Corynebacterium glutamicum gene, encoding CAT cDNA, was cloned into pIVEX1.3, between the NcoI and SmaI restriction sites. A PCR product containing the complete coding region of cycle 3 GFP (4) was similarly cloned into pIVEX2.2, between the NcoI and SmaI restriction sites. The sequences of all expression plasmids were verified by DNA sequencing.

\section{Purification and Quantification of GFP-Lunasin and IL-2}

To generate sufficient protein for bioanalysis, GFP-lunasin fusion protein was produced in 10 separate $(1 \mathrm{~mL})$ expression reactions. The contents of the reaction compartments were pooled and purified using a Ni-NTA column (Qiagen, Hilden, Germany). The concentration of purified GFP-lunasin was estimated using SDS-PAGE, with a comparison against a known concentration of purified, recombinant GFP (Roche Molecular Biochemicals). IL-2 was also purified using Ni-NTA column chromatography. The concentration of purified IL-2 was estimated by ELISA (Bender MedSystems, Vienna, Austria).

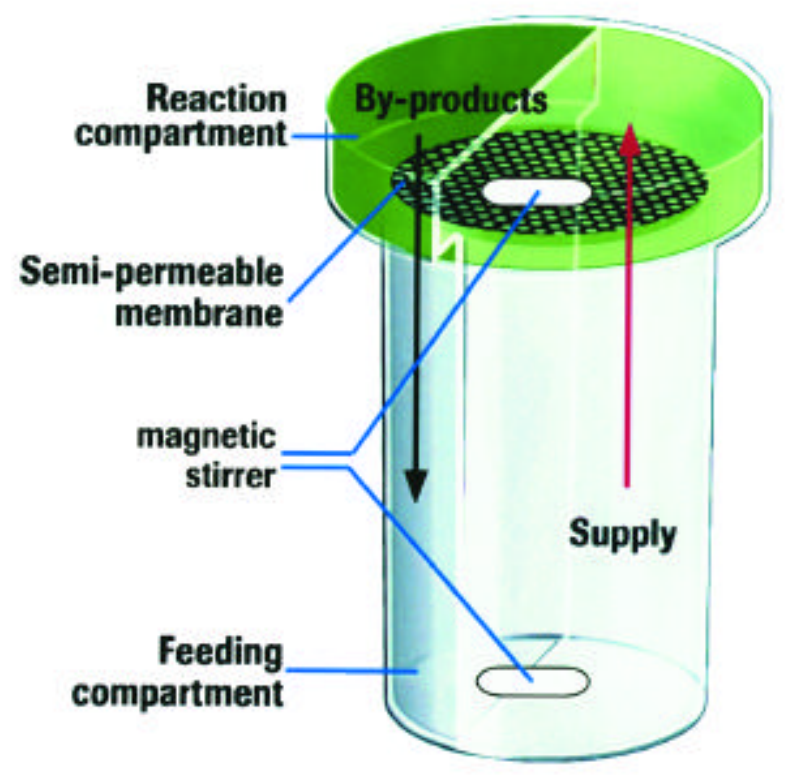

Figure 1. A schematic representation of the principle of continuous-exchange cell-free technology utilized by the RTS 500. The 1-mL Reaction Compartment (green) contains a reconstituted, lyophilized lysate fromE. coli containing the translation machinery, plasmid DNA encoding the gene of interest, T7 RNA polymerase to produce RNA from the DNA template, as well as energy and feeding components. The lower, 10-mL Feeding Compartment (white) contains energy and feeding components. The two compartments were separated by a semi-permeable membrane (patterned), and each contains a stir bar to ensure the proper movement of small molecules across the membrane. 


\section{Biological Activity of GFP-Lunasin Fusion Protein Using} NIH 3 T3 Focus-Forming Assay

To verify the biological activity of the lunasin fusion protein, we compared the ability of GFP-lunasin to inhibit foci formation to lunasin, a chemically synthesized 40-amino-acid peptide (Reference 7 and unpublished data). NIH 3T3 cells were plated at a density of $7 \times 10^{4}$ cells/well on a 6 -well plate. On day 2, $250 \mathrm{ng}$ mutant H-ras (G12V) plasmid DNA per well was delivered by transfection. After transfection, there were equal molar concentrations $(1 \mu \mathrm{M})$ of chemically synthesized lunasin and GFP-lunasin fusion protein. PBS was used instead of lunasin in the control wells. On day 4, the cells were split 1:6. Each well of transfected cells was transferred into six new wells. The media was changed as needed; however, no additional lunasin was added after day 4. On day 21 , the cells were fixed and stained with crystal violet, and the foci were counted.

\section{Functional Activity Testing of IL-2 through the Generation of LAK Cells}

The biological activity of IL-2 produced using the RTS 500 was assessed by its ability to stimulate lymphokine-activated killer (LAK) cell killing. LAK activity was determined using a standard 4-h ${ }^{51} \mathrm{Cr}$-release assay (3). The natural killer (NK)-resistant Burkitt lymphoma cell line, Daudi (ATCC no. CCL243), was used as the target. The amount of active IL-2 was determined by a comparison with an IL-2 standard (Shionogi, Osaka, Japan).

\section{Western Blot Analysis of Co-Expressed GFP and CAT}

Equal amounts of plasmid DNA, encoding CAT and GFP, were added for both multiple plasmids $(7.5 \mu \mathrm{g} /$ plasmid $)$ and individual plasmids $(15 \mu \mathrm{g})$ to an RTS 500 reaction. After 24 $\mathrm{h}$, the proteins were analyzed by western blotting. Immobilized GFP and CAT were both detected using anti-His6 antibody, followed by anti-mouse IgG conjugated to alkaline phosphatase. They were then visualized using NBT/BCIP as the substrate. Western blot detection reagents were provided by Roche Molecular Biochemicals.

\section{RESULTS AND DISCUSSION}

The RTS 500 was used in this study to successfully express two functional proteins that are either impossible or difficult to obtain in preparative quantities from cell-based expression systems. Studies of the biological function of lunasin have previously only been possible through the chemical synthesis of the polypeptide (6). The fact that $270 \mu \mathrm{g}$ lunasinGFP fusion protein were easily expressed and purified from $10 \mathrm{~mL}$ pooled RTS 500 reaction-compartment contents (Figure 2A) illustrates a major advantage of the RTS 500 over cell-based expression (when expressing toxic proteins). Figure 2 shows the majority of GFP-lunasin fusion protein was produced in the soluble fraction; however, some insoluble product was present. NIH 3T3 cells, treated with purified GFP-lunasin, showed a $50 \%$ reduction in the number of oncogenic ras-induced foci. This result was comparable, on a mole-to-mole basis, with the result obtained using a chemically synthesized 40-amino-acid residue (i.e., lunasin polypeptide) (Figure 2B). Other cell-toxic proteins, such as MID1 (microtubule-binding protein) (personal communication, A. Luckling, Max-Plank Institute), have also been expressed using the RTS 500.

Solubility problems are commonly encountered when attempting to express proteins in E. coli. For example, human IL-2 requires tedious optimization of solubilization and refolding processes to obtain a soluble, active protein. Insoluble IL-2 produced in $E$. coli contains inappropriately formed disulfide bonds that must be reduced and reformed under precisely controlled conditions. The RTS 500 is more reducing environment for cysteine than intact $E$. coli cells. This may prevent the formation of inappropriate disulfide bonds and results in increased solubility of IL-2 in the RTS 500. Very little optimization was needed to produce soluble human IL-2 in the RTS 500 with yields up to $600 \mu \mathrm{g} / \mathrm{mL}$ (Figure 3A). The material stimulated the generation of LAK activity, with a bioactivity that was nearly identical to commercially available IL-2 standard produced in bacteria (Figure 3C). Rapid optimization of human IL-2 expression was easily achieved because of the "open" nature of the RTS 500. The amount of protein produced could be easily monitored by obtaining samples (at anytime during the reaction) from the upper compartment of the RTS 500. When adding different amounts of plasmid, the amount of soluble IL-2 protein produced reached

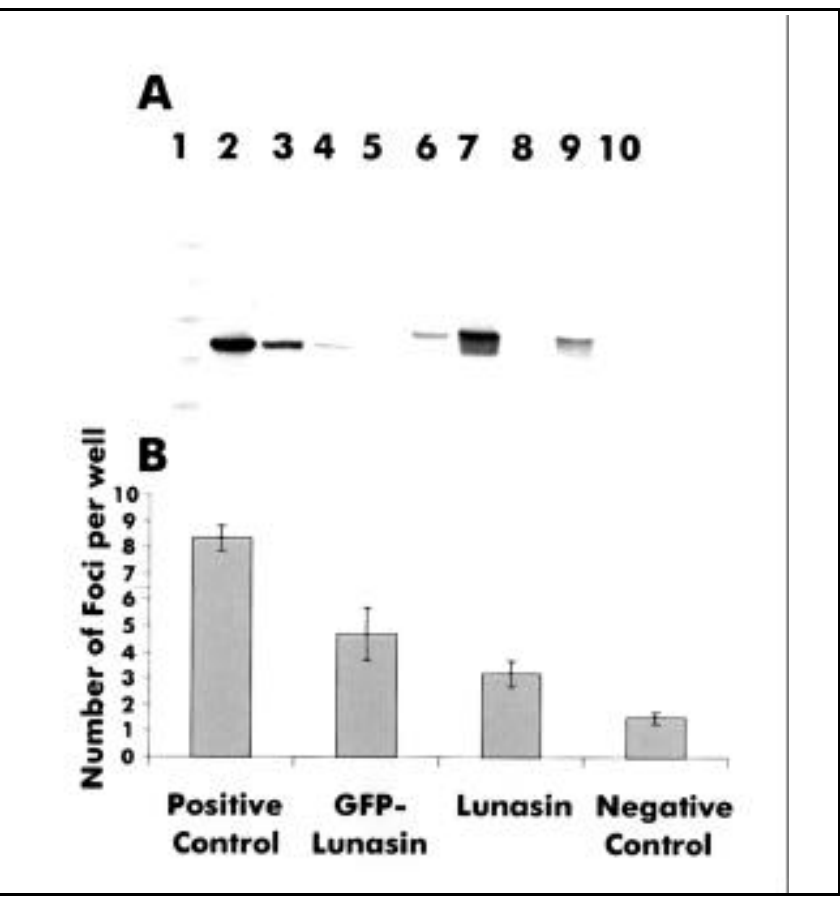

Figure 2. Production and functional analysis of GFP-lunasin fusion protein. (A) Western blot analysis of rGFP and GFP-lunasin fusion protein produced in the RTS 500 with an anti-GFP antibody. Lane 1, molecular weight marker (Invitrogen, Carlsbad, CA, USA). Lanes 2-5 contain 50, 10, 5, and 1 ng purified rGFP, respectively. Lane $6,2 \%$ of the insoluble fraction of a lunasin-GFP expression; lane 7, $2 \%$ of the soluble fraction of a lunasin-GFP expression; lane $8,1 \%$ of the insoluble fraction of a lunasin-GFP expression; lane $9,1 \%$ of the insoluble fraction of a lunasin-GFP expression; and lane 10, $0.2 \%$ of the soluble fraction of a lunasin-GFP expression. (B) Lunasin functional analysis. Reduction in the number of ras-induced foci in NIH $3 \mathrm{~T} 3$ cells by treatment with an equal molar concentration of a GFP-lunasin produced in the RTS 500 or a chemically synthesized lunasin peptide. The GFP-lunasin fusion protein inhibited the ras-induced formation of foci similarly to the chemically synthesized 21 -amino-acid residue polypeptide. Negative control contains no ras, and the positive control contains no lunasin. 
a plateau at about $15 \mu \mathrm{g}$ plasmid DNA (Figure 3A); however, there may have been a slight reduction of productivity at 20 $\mu \mathrm{g}$. Figure $3 \mathrm{~B}$ shows the optimal temperature for the expression of IL-2 to be $30^{\circ} \mathrm{C}$. Expression at $37^{\circ} \mathrm{C}$ caused a marked decrease in IL-2 production. The total yield of protein reached a plateau at $24 \mathrm{~h}$. Although soluble IL-2 was produced in the RTS 500 using the standard reaction mixture, other insoluble proteins produced in solution by adding different solublization reagents. For example, murine endostatin, an insoluble protein when expressed in E. coli $(16,22)$, has been successfully expressed as a soluble protein using this system and adding purified $E$. coli chaperones to the reaction.

While cellular systems are capable of expressing multiple proteins in a single experiment, it is much easier to accom plish this in an in vitro transcription/translation system. To express multiple proteins from different plasmids in bacteria, each plasmid must encode a different antibiotic resistance marker and have compatible origins of replication (11). In contrast, multiple plasmids may simply be added to the reaction mixture when using the RTS 500. Using $15 \mu \mathrm{g}$ plasmid and optimized conditions, GFP alone was typically expressed at $600 \mu \mathrm{g} / \mathrm{mL}$ reaction, while under the same conditions, CAT produced $900 \mu \mathrm{g} / \mathrm{mL}$. However, when GFP was co-expressed with CAT ( 7.5 or $15 \mu \mathrm{g}$ each plasmid), only the expression of GFP was reduced, relative to GFP expressed alone. The amount of CAT expressed during co-expression with GFP was remarkably similar to the amount CAT expressed alone (Figure 4A). A reduction in the yield of protein is often observed when expressing multiple proteins using the RTS 500.
Although only two plasmids were added simultaneously in our example, many plasmids could theoretically be expressed in a single experiment. When co-expressing multiple proteins with the RTS 500, there may be a reduction in the total amount of an individual protein produced, relative to that same protein expressed alone. However, we have also observed that when only $0.5 \mathrm{~mL}$ reaction mixture was added into the $1-\mathrm{mL}$ reaction compartment, the amounts of functional GFP and CAT increased. This was probably due to better aeration and oxidization conditions. Researchers can take advantage of the system's openness to adjust the reaction components, such as amount and ratio of the individual plasmids and co-factors, to balance the expression levels.

$\beta$-galactosidase is a large homo-tetrameric protein com posed of four identical 116-kDa subunits and requires correct assembly for functional activity. This approximately 150 $\mu \mathrm{g} / \mathrm{mL}$ functional $\beta$-galactosidase has been expressed using this system. This data, together with the co-expression data generated in this study, would suggest that the system might be useful for protein-protein interaction studies and possibly allow for the in vitro assembly of hetero-multimeric proteins.

Other researchers have successfully produced eukaryotic and prokaryotic proteins from a wide number of species using the RTS 500 E. coli Circular Template System. The RTS 500 system has produced proteins as large as a $120-\mathrm{kDa}$ DNA polymerase and as small as the cytomegalovirus (CMV) small capsid protein $(9.5 \mathrm{kDa})$. An extensive listing of these and other proteins successfully expressed using the RTS 500 is maintained at http://www.proteinexpression.com. This list also in-

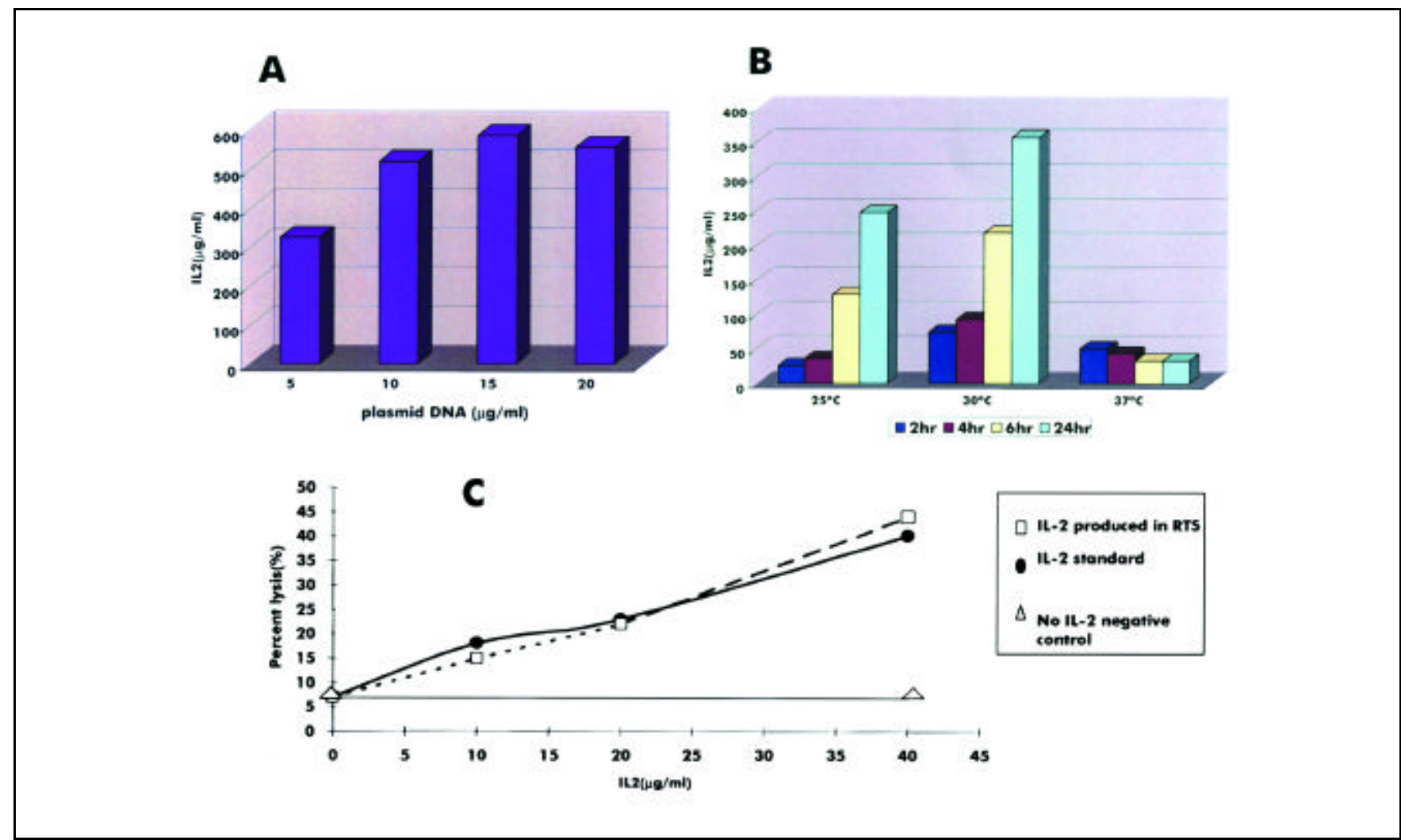

Figure 3. Optimization of expression conditions and functional analysis of IL-2. (A) Recombinant human IL-2 was produced using the RTS 500 system at $30^{\circ} \mathrm{C}$ for $24 \mathrm{~h}$ with increasing amounts of pIVEX2.4-IL2 plasmid DNA. The amount of IL-2 was determined by ELISA. (B) RTS expressions were performed with $15 \mu \mathrm{g}$ plasmid DNA at three different temperatures. The reaction chambers were sampled at the indicated times. The amount of IL-2 was determined by ELISA. (C) IL-2 functional analysis. Percent killing of Daudi cells by LAK cells stimulated with IL-2 produced either in a cell-based bacterial system (closed circles) or in the RTS 500 (open squares). No IL-2 was included as negative control (open triangles). 


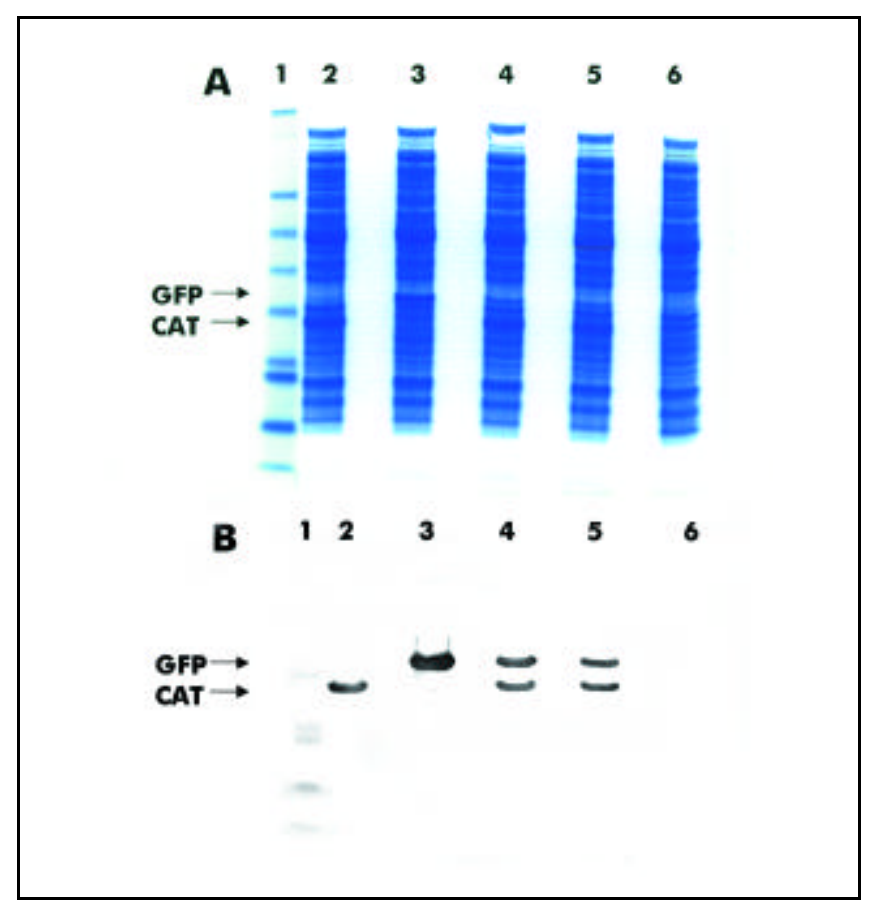

Figure 4. Co-expression of GFP and CAT in the RTS 500 system. Plasmids encoding CAT and GFP were added either singly or in combination to the RTS 500 system. After $20 \mathrm{~h}$ at $30^{\circ} \mathrm{C}$, the reactions were assayed. (A) Coomassie ${ }^{\circledR}$-stained SDS-PAGE. (B) Western blot analysis with anti-His antibodies. Lane 1, See-Blue ${ }^{\mathrm{TM}}$ pre-stained standard (Invitrogen); lane 2, expression of CAT alone with $15 \mu \mathrm{g}$ plasmid; lane 3 , expression of GFP alone with $15 \mu \mathrm{g}$ plasmid; lane 4, GFP and CAT in the same reaction with $7.5 \mu \mathrm{g}$ plasmid each; lane 5, GFP and CAT in the same reaction with $15 \mu \mathrm{g}$ plasmid each; and lane 6, RTS lysate without plasmid as a negative control.

cludes proteins that were expressed with much lower yields than those discussed in this paper. It may be necessary to create optimized conditions for generating soluble and correctly folded proteins by including additives such as detergents, protease inhibitors, small molecules, and purified auxiliary proteins to the Rapid Translation System during protein synthesis. Conditions have also been identified that result in successful expression of functionally active recombinant plasminogen activator (rPA). This process requires the correct formation of nine disulfide bonds (18). During these studies, the RTS 500 demonstrated itself as a powerful tool for protein expression.

\section{ACKNOWLEDGMENTS}

The authors gratefully acknowledge the contributions of Katrin Zaiss, Zane Liu, J.J. Liu, and Stacey Traviglia.

\section{Note Added in Proof:}

Following the submission of this manuscript, enhancements in the energy regeneration biochemistry of the RTS 500 have led to significant increases in the yield of most proteins. The new RTS 500 E. coli HY Kit still takes advantage of CECF technology, but the new energy regeneration chemistry results in 5- to 10 -fold higher yields of most protein compared to the original RTS 500 E. coli Circular Template Kit evaluated in this paper. Current information regarding all RTS Kits and expression data may be found online at http://www.proteinexpression.com.

\section{REFERENCES}

1.Ambion. 1998. Translation: flexibility and high yields. TechNote 5:5.

2.Baranov, V.I., I.J. Morozov, S.A. Ortlepp, and A.S. Spirin. 1989. Gene expression in a cell-free system on the preparative scale. Gene 84:463466.

3.Coligan, J.E., A.M. Kruisbeek, D.H. Margulies, E.M. Shevach and W. Strober (Eds.). 1992. Current Protocols in Immunology. Greene Publishing Associates and Wiley-Interscience Publishers, New York.

4.Crameri, A., E.A. Whitehorn, E. Tate, and W.P.C. Stemmer. 1996. Im proved green fluorescent protein by molecular evolution using DNA shuffling. Nat. Biotechnol. 14:315-319.

5.Endo, Y., S. Otsuzuki, K. Ito, and K. Miura. 1992. Production of an enzymatic active protein using a continuous flow cell-free translation system. J. Biotechnol. 25:221-230.

6.Galvez, A.F. and B.O. de Lumen. 1999. A soybean cDNA encoding a chromatin-binding peptide inhibits mitosis of mammalian cells. Nat. Biotechnol. 17:495-500.

7.Garte, S.J., D.D. Currie, and W. Troll. 1987. Inhibition of H-ras oncogene transformation of NIH 3 T3 cells by protease inhibitors. Cancer Res. 47:3159-3162

8. Guisez, Y., J. Demolder, N. Mertens, A. Raeymaekers, G. Plaetinck, J. Robbens, J. Vandekerckhove, E. Remaut, and W. Fiers. 1993. Highlevel expression, purification, and renaturation of recombinant murine interleukin-2 from E. coli. Protein Expr. Purif. 4:240-246.

9.Hora, M.S., R.K. Rana, C.L. Wilcox, N.V. Katre, P. Hirtzer, S.N. Wolfe, and J.W. Thomson. 1992. Development of a lyophilized formulation of interleukin-2. Dev. Biol. Stand. 74:295-303.

10.Jermutus, L., L.A. Ryabova, and A. Pluckthun. 1998. Recent advances in producing and selecting functional proteins by using cell-free translation. Curr. Opin. Biotechnol. 9:534-548.

11.Khokhlatchev, A., S. Xu, J. English, P. Wu, E. Schaefer, and M.H. Cobb. 1997. Reconstitution of mitogen-activated protein kinase phosphorylation cascades in bacteria. J. Biol. Chem. 272:11057-11062.

12.Kigawa, T. and S. Yokoyama. 1991. A continuous cell-free protein synthesis system for coupled transcription and translation. J. Biochem. 110:166-168.

13.Kigawa, T., Y. Muto, and S. Yokoyama. 1995. Cell-free synthesis and acid selective stable isotope labeling of proteins for NMR analysis. J. Biomol. NMR 6:129-134.

14.Kim, D.-M. and C.-Y. Choi. 1996. A semicontinuous prokaryotic coupled transcription/translation system using a dialysis membrane. Biotechnol. Prog. 12:645-649.

15.Kim, D. and J.R. Swartz. 1999. Prolonging cell-free protein synthesis with a novel ATP regeneration system. Biotechnol. Bioeng. 66:180-188.

16.Nishihara, K., M. Kanemori, H. Yanagi, and T. Yura. 2000. Overexpression of trigger factor prevents aggregation of recombinant proteins in Escherichia coli. Appl. Environ. Microbiol. 66:884-889.

17. Rattan, S.I. and O. Kristensen. 1990. Continuous gene expression in vitro: the Spirin system. Trends Biotechnol. 8:275-276.

18. Renatus, M., R.A. Engh, M.T. Stubbs, R. Huber, S. Fischer, U. Kohnert, and W. Bode. 1997. Lysine 156 promotes the anomalous proenzyme activity of tPA: X-ray crystal structure of single-chain human tPA. EMBO J. 16:4797-4805.

19.Spirin, A.S., V.I. Baranov, L.A. Ryabova, S.J. Ovodov, and J.B. Alakhov. 1988. A continuous cell-free translation system capable of producing polypeptides in high yield. Science 242:1162-1164.

20.Stiege, W. and V.A. Erdmann. 1995. The potentials of the in vitro protein biosynthesis system. J. Biotechnol. 41:81-90.

21.Weir, M.P., J. Sparks, and A.M. Chaplin. 1987. Micropreparative purification of recombinant human interleukin-2. J. Chromatogr. 396:209215.

22.You, W.K., S.H. So, H. Lee, S.Y. Park, M.R. Yoon, S.I. Yoon, S.I. Chang, H.K. Kim et al. 1999. Purification and characterization of recombinant murine endostatin in E. coli. Exp. Mol. Med. 31:197-202.

Received 1 March 2001; accepted 2 May 2001.

Address correspondence to:

George A. Martin

Roche Molecular Biochemicals

2929 Seventh Street, Suite 100

Berkeley, CA 94710, USA

e-mail: george.martin@roche.com 